\title{
Preanthesis biomass accumulation of plant and plant organs defines yield components in wheat
}

\author{
Quan Xie, Sean Mayes, Debbie L. Sparkes* \\ Division of Plant and Crop Sciences, The University of Nottingham, Sutton Bonington
}

Campus, Loughborough, Leicestershire LE12 5RD, UK

*Corresponding author.

Tel.: +44 1159516074

Fax: +44 1159516060

E-mail address: debbie.sparkes@nottingham.ac.uk (Debbie L. Sparkes)

Abbreviations: ADM, accumulated dry matter; CGR, crop growth rate; cM, centi-Morgan; ${ }^{\circ} \mathrm{Cd}$, degree days; GS39, Growth Stage 39 (the time at full flag leaf emergence); HI, harvest index; $\mathrm{H}^{2}$, broad sense heritability; LI, light interception; LOD, logarithm of the odds; QTL, quantitative trait loci; RIL, recombinant inbred line; RUE, radiation use efficiency; SFI, spike fertility index; SGD, spike growth duration; SGR, spike growth rate; SPI, spike partitioning index; TGW, thousand grain weight; WSC, water soluble carbohydrate.

\section{Artwork information}

Fig. 1 in black and white, 2-column, portrait

Fig. 2 in black and white, 2-column, portrait

Fig. 3 in black and white, single column, portrait

Fig. 4 in colour in print, 2-column, landscape 


\section{Abstract}

The preanthesis period in wheat is critical for growth of plant organs including leaves, stems, spikes and roots. However, the roles of the preanthesis biomass accumulation of plant and plant organs in yield determination are only partially elucidated, and the underlying genetic basis remains largely unknown. This study aimed to understand the physiological and genetic relationships between preanthesis biomass accumulation and yield determination. In a mapping population of bread wheat (Triticum aestivum 'Forno') and its relative spelt (Triticum spelta 'Oberkulmer') contrasting for biomass, the dry weight of above-ground whole shoots and different organs, and leaf area, were analysed at GS39 (full flag leaf emergence) and anthesis. Yield components (thousand grain weight, grains per spike, final shoot biomass and grain weight per spike) and plant height were measured at maturity, followed by identification of quantitative trait loci (QTL) for all above traits. Field experiments were carried out in UK in 2011-2012 and 2012-2013 seasons, each using a randomised complete block design with three replicates. The results showed that there was a significant variation in biomass and its partitioning to organs at different stages. Consistent with the previous findings, stem water soluble carbohydrates and spike dry weight at anthesis contributed to thousand grain weight and grains per spike, respectively. In addition, this study revealed many other traits positively associated with one or more yield components, including biomass and leaf area at GS39, leaf and structural stem growth as well as whole shoot biomass at anthesis, and higher dry matter accumulation and crop (and spike) growth rates between the two stages. Increasing shoot biomass by removing other tillers at GS39 led to higher grain number and grain weight per spike. These results indicate the importance of the preanthesis growth of plant and plant organs for yield determination. Plant height was only weakly correlated with final biomass at maturity so it is possible to produce high-biomass genotypes without increasing plant height. Genetic analysis revealed 193 QTL associated with 
biomass and biomass-related traits. Frequent QTL coincidences between biomass and yield traits were observed, mainly on chromosomes $2 \mathrm{~B}, 3 \mathrm{~A}, 4 \mathrm{~A}, 4 \mathrm{~B}, 5 \mathrm{~A}, 6 \mathrm{~A}$ and $7 \mathrm{~B}$, indicating pleiotropy or tight gene linkages, consistent with their phenotypic associations. The preanthesis biomass traits associated with yield components and the underlying QTL, would facilitate the trait-based physiological and molecular breeding in wheat.

Keywords: Bread wheat; Spelt; Biomass; Plant organ; Yield component; Quantitative trait locus 


\section{Introduction}

Wheat (Triticum aestivum L.) alone provides $19 \%$ of the calories and $20 \%$ of the protein for human diets (Braun et al., 2010), and hence is a key contributor to global food security. A substantial increase in wheat production is required to keep pace with the burgeoning world population, being over 9 billion by 2050, as projected by the United Nations. However, the annual growth rates of wheat production and yield have slowed down, that is, only around $1.0 \%$ for both in last decades, less than those of demand (1.7\%) (Reynolds et al., 2012; Ray et al., 2013). Future yield gain has also been challenged by global climate change, diminishing natural resources, rising prices for fertilisers and pesticides, and competition for arable land (Reynolds et al., 2012). From a breeding perspective, wheat cultivars need to be improved for further genetic gain. Conventional breeding has been mainly based on grain yield per se, together with the resistance to lodging, and biotic and abiotic stresses. Such a strategy can be substantially enhanced by understanding the physiological and genetic basis of yield. Given its complexity, wheat grain yield can be dissected into relatively simpler traits: grain number and individual grain weight (numerical components), or biomass and harvest index (HI, biomass partitioning to grains) (physiological components). Traits influencing these components during plant growth and development have to be clarified so that the pathways of yield determination can be understood. Favourable traits and their underlying genes will be assembled to form ideotypes for wheat breeding. This strategy, i.e. trait-based physiological and molecular breeding, is more fundamental for yield improvement (Foulkes et al., 2011).

For numerical components, yield progress has been highly associated with an increase in grain number rather than individual grain weight (Slafer and Andrade, 1993; Shearman et al., 2005; Sanchez-Garcia et al., 2013). In some regions, grain weight has also been improved and contributed most to yield gain, especially in recent decades (Sadras and Lawson, 2011; Wu et al., 2014). Grain number is mainly determined by the preanthesis floret survival within 
spikelets. Each spikelet produces up to ten florets, but fewer than five (mainly those closest to rachis) can set grains, and the remaining ones die just before anthesis (Kirby, 1988; GonzálezNavarro et al., 2015). It seems that the proportion of fertile florets, rather than the total number of floret primordia, is the main factor that determines the final number of fertile florets at anthesis (Brooking and Kirby, 1981; González-Navarro et al., 2015). There is a strong and positive relationship between spike dry weight (DW) at anthesis and floret survival (Fischer, 1985, 2011; González et al., 2011). As spike DW can be expressed as a function of spike growth duration (SGD), crop growth rate (CGR), and biomass partitioning to spikes (spike partitioning index, SPI), an increase in these traits during the critical period before anthesis would favour spike growth and in turn floret survival (Fischer, 2011; Garcia et al., 2014). On the other hand, biomass partitioning to stems should be decreased to minimise the competition between spikes and stems for assimilates (Kirby, 1988).

Individual grain weight is largely determined during grain filling, but a short period before anthesis is also important. It has been proposed that carpel size prior to anthesis may set an upper limit for grain development, as there is a strong and positive relationship between them (Calderini et al., 1999; Hasan et al., 2011; Xie et al., 2015). Carpel growth seems to be responsive to preanthesis biomass accumulation and partitioning; for example, increasing assimilate availability through de-graining at heading leads to greater carpel size (Calderini and Reynolds, 2000). After fertilisation, there is evidence that endosperm cell number, as affected by the assimilate availability during the first two weeks after anthesis, defines a threshold of potential grain weight (Brocklehurst, 1977; González et al., 2014). Rapid dry matter accumulation is then initiated, followed by grain maturation and desiccation. During grain filling, the assimilates are supplied from the current photosynthesis and dry matter translocation of leaves, spikes and stems. The amount of newly synthetic biomass between anthesis and maturity depends on the timing of plant senescence, and delayed senescence is 
usually believed favourable (Gregersen et al., 2013). Water soluble carbohydrates (WSCs) in vegetative parts (mainly stems) show positive associations with grain weight and yield (Foulkes et al., 2007; Rebetzke et al., 2008), and the estimated contribution of total WSCs to yield can be as high as $50 \%$, depending on growing conditions (e.g. more significant under drought) (van Herwaarden et al., 1998a; Rebetzke et al., 2008). In addition, structural nutrients (particularly nitrogen) can be partly recycled during terminal senescence for growing grains (Distelfeld et al., 2014).

Physiologically, yield is a product of plant biomass and HI. HI has been largely improved with the use of dwarfing genes, and closely associated with yield progress (Shearman et al., 2005; Sadras and Lawson, 2011; Sanchez-Garcia et al., 2013). HI currently reaches approximately $0.45-0.50$ in spring wheat and $0.50-0.55$ in winter wheat, approaching its theoretical maximum value (approximately 0.64 in winter wheat) (Foulkes et al., 2011; Reynolds et al., 2012). To further increase HI, biomass partitioning to different plant organs needs to be optimised. Genetic variation in biomass partitioning has been observed in elite wheat lines, and this variation can be broadened by introducing desirable traits from wild species existing in Triticeae (Foulkes et al., 2011; Reynolds et al., 2012). However, given no systematic progress in HI since the early 1990s, future yield gain will depend more on an increase in biomass (Fischer, 2011; Reynolds et al., 2012). Recent yield improvement has showed an association with increased biomass (Shearman et al., 2005; Sadras and Lawson, 2011). Biomass is a function of light interception (LI) and radiation use efficiency (RUE, biomass per unit of radiation intercepted) (Reynolds et al., 2012). LI can be improved by optimising canopy size (e.g. large leaves and spikes), architecture (e.g. erect leaves) and longevity (e.g. early vigour and late senescence), while increasing plant photosynthesis is required for higher RUE (Reynolds et al., 2012). 
As stated above, there are quite a number of studies focusing on the pre-anthesis phase in wheat, including stem elongation period and spike dry weight at anthesis as related to floret fertility, and carpel size and stem WSCs as related to individual grain weight. However, whether the other traits such as leaf growth, structural stem development, spike growth dynamic, competition between plant parts and overall crop growth before anthesis, would determine the process of yield formation is rarely elucidated in detail. Furthermore, little is known about the underlying genetic elements bridging the preanthesis plant growth with yield production. This work aimed to provide a comprehensive understanding of the physiological and genetic associations of the preanthesis growth of plant and plant organs with yield determination. Plant-level biomass accumulation and partitioning at key growth stages (GS), namely GS39 (full flag leaf emergence), anthesis and maturity, were analysed in a mapping population of bread wheat (T. aestivum 'Forno') and spelt (T. spelta 'Oberkulmer') contrasting for biomass production. Although observation of biomass in this study was based on single-plant level rather than crop scale, these results help to elucidate formation of yield components in terms of physiology and genetics in a simpler way. The physiological traits of biomass associated with yield components were determined, and subsequently the genetic dissection of biomass, biomass-related traits, and yield components, were carried out via a detailed quantitative trait locus (QTL) analysis. 


\section{Materials and Methods}

\subsection{Plant materials and field experiments}

Spelt is a relative of bread wheat, and has higher biomass but lower harvest index than the latter (Koutroubas et al., 2012). A cross between the Swiss winter bread wheat 'Forno' and Swiss winter spelt 'Oberkulmer' was carried out to introduce genetic variation in biomass, and a total of $226 \mathrm{~F}_{5}$ recombinant inbred lines (RILs) were derived (Messmer et al., 1999). This population was grown in a sandy loam soil $(\mathrm{pH} 7.6, c .80$ and c. $70 \mathrm{~kg}$ nitrogen ha-1 available in $0-90 \mathrm{~cm}$ depth as measured before the growing seasons in 2011 and 2012, respectively) at University of Nottingham Farm, Leicestershire, UK. Field experiments were conducted in 2011-2012 and 2012-2013 (referred hereafter as 2012 and 2013, respectively), and arranged according to a randomised complete block design with three replicates. The seeds were sown at 250 seeds $\mathrm{m}^{-2}$ in plots of $6 \times 1.6 \mathrm{~m}$ in 2011 and $12 \times 1.6 \mathrm{~m}$ in 2012. An additional c. 140 (in 2012) and c. 160 (in 2013) $\mathrm{kg}$ nitrogen $\mathrm{ha}^{-1}$ in the form of ammonium nitrate prills were applied (three splits: early March, April and May). When necessary, fungicides, insecticides and herbicides were also applied to maintain undisturbed healthy crop growth. For phenotyping, two subsets of the whole mapping population were selected, including 72 RILs in 2012 and 110 RILs in 2013, based on their similar flowering time, which ensured that the lines would grow under similar environmental conditions.

\subsection{Biomass, leaf area, yield components and plant height}

Biomass and its partitioning to different plant organs were assessed at key growth stages, namely GS39 (full flag leaf emergence), GS61 (anthesis), and GS92 (maturity) (Zadoks et al., 1974). GS39 was selected because it is the onset of rapid spike growth, and because it represents the time of full flag leaf emergence so measurements of flag leaf area and dry weight are reliable and can be compared among genotypes. In 2012, a central area of $0.5 \times 0.5$ $\mathrm{m}$ from each plot of the subsets was harvested at GS39. A c. $20 \%$ sub-sample of shoots was 
separated randomly after cutting at ground level, and the fertile shoots were counted. The shoots were then partitioned into leaves, and stems plus sheaths (referred hereafter as stems). Total leaf area was measured using an area meter (LI3100, LI-COR, USA). Each part was then dried in an oven at $85^{\circ} \mathrm{C}$ for $48 \mathrm{~h}$, and weighed. Dry weight per shoot (excluding roots) and biomass partitioning to different plant organs (absolute weight and percentages) were calculated. Meanwhile, five main shoots from each plot were also sampled exactly at GS39, by daily observation for the growth stages of individual RILs. The spikes were collected by dissecting stems, and then the DW of spikes and remaining shoots was recorded. At anthesis, five main shoots from each plot were harvested exactly at GS61 (the first anthers just visible), and partitioned into flag and remaining leaves, stems, and spikes. Leaf area was measured, and then each organ was oven-dried immediately at $85^{\circ} \mathrm{C}$ for $48 \mathrm{~h}$. To measure stem WSC content at anthesis, the dried stems were finely ground by a mill. Carbohydrates were extracted in $80 \%$ ethanol and water, following the method of van Herwaarden et al. (1998a). WSC content in the extracts was determined using the anthrone method of Yemm and Willis (1954). Stem WSC content per shoot was calculated, and structural stem DW was the difference between total stem and WSC weight. The ratio of spike to structural stem DW (spike:structural stem) was obtained. At maturity, a central area of $0.5 \times 0.5 \mathrm{~m}$ from each plot was harvested. A c. $20 \%$ sub-sample of above-ground shoots was counted for fertile shoots, and partitioned into leaves, stems, and spikes. The DW of each organ was recorded after drying at $85^{\circ} \mathrm{C}$ for $48 \mathrm{~h}$. The spikes were then threshed, and the total grain and chaff weight was obtained. In 2013, 10, 10 and 20 main shoots were sampled exactly at GS39, anthesis, and maturity, respectively, and measurements on biomass and leaf area followed the procedures as described above. In terms of yield components, grain weight per spike, grains per spike, thousand grain weight (TGW), dry weight (final biomass) per shoot and HI were adopted from a previous study based on the same field experiments (using the same RILs 
grown in the same seasons) (Xie et al., submitted). Briefly, grain weight per spike was calculated as the total grain weight divided by shoot number in the sub-samples at maturity. A sample of 250 grains was counted and weighed to calculate TGW, and grains per spike were computed from grain weight per spike and TGW. Dry weight per shoot was the sum of DW of all organs at maturity, and HI was the total grain weight divided by total biomass. Spike fertility index (SFI) was calculated as the grains per spike divided by spike DW at anthesis. For plant height, five main shoots from each plot were measured from stem bases to spike tips (excluding awns) at maturity.

Based on the above data, accumulated dry matter (ADM) of the whole shoots from GS39 to anthesis and from anthesis to maturity was obtained. For each period, CGR was calculated from the ADM divided by the accumulated thermal time (degree days, ${ }^{\circ} \mathrm{Cd}$; base temperature $0^{\circ} \mathrm{C}$ ). To determine the initiation of spike growth, five main shoots from each plot were collected approximately at GS33 (the third node of stem detectable) in both years. The spikes were removed by opening the sheaths, oven-dried, and weighed. Spike growth rates (SGR) from GS33 to GS39, and from GS39 to anthesis, were calculated as the differences in spike DW divided by accumulated thermal time during each period.

\subsection{De-tillering at GS39}

Ten central plants in each plot of five well-established RILs were selected randomly in 2013. All tillers of these plants were removed in order to allow the remaining main shoots to grow under relatively saturated resource supply. Ten main shoots from intact plants were used as control. De-tillering and control shoots were tagged, harvested and processed at maturity. TGW, grains per spike, dry weight per shoot, HI and grain weight per spike were calculated.

\subsection{De-graining at anthesis}

The whole subset (110 RILs) was used for source-sink manipulation during grain filling in 2013. Five main spikes at GS61 were selected from each plot, and all spikelets along one side 
of each spike were trimmed, potentially doubling the source capacity for the remaining grains (Slafer and Savin, 1994). The trimmed spikes, together with five intact spikes as control, were collected, threshed, oven-dried, and weighed. Individual grain weight in trimmed and intact spikes was defined as potential (PGW) and actual grain weight (AGW), respectively. The difference between PGW and AGW was considered as extra assimilate use. Ten grains from each of ten RILs contrasting for grain weight were measured for grain dimensions (length, width and height) using an electronic calliper (OD-15CP, Mitutoyo, UK). Grain volume was calculated using the formula: $V_{\mathrm{g}}=(4 / 3) \pi a b c$, where $V_{\mathrm{g}}=$ grain volume, $\pi=3.1416, a=0.5$ grain length, $b=0.5$ grain width, and $c=0.5$ grain height (Hasan et al., 2011). Grain density was calculated from grain weight and volume.

\subsection{Statistical analysis of phenotypic data}

Analysis of variance was carried out to test the genetic variation in all traits among the RILs. Pearson correlations and regression analysis were used to determine the relationships between different traits. Phenotypic data were transformed by square root, logarithmic or arcsine methods as necessary to improve their normality. Broad sense heritability $\left(\mathrm{H}^{2}\right)$ of each trait across years was computed as: $H^{2}=\sigma_{\mathrm{g}}^{2} /\left(\sigma_{\mathrm{g}}^{2}+\sigma_{\mathrm{ge}}^{2} / n+\sigma_{\mathrm{e}}^{2} / r n\right)$, where $\sigma_{\mathrm{g}}^{2}$ is the genotypic variance, $\sigma_{\mathrm{ge}}^{2}$ is the genotype-by-environment interaction variance, $\sigma_{\mathrm{e}}^{2}$ is the error variance, $n$ is the number of environments (years), and $r$ is the number of replicates per environment. Variance components were estimated by residual maximum likelihood (REML) in the linear mixed model, considering year and replicate as fixed factors, and genotype and genotype-byyear interaction as random factors. Statistical analyses were performed using Genstat v17 and GraphPad Prism v6.05.

\subsection{QTL analysis}

The genetic map of Forno $\times$ Oberkulmer can be found in the GrainGenes database (http://wheat.pw.usda.gov/GG2/index.shtml). This map consists of 182 polymorphic markers 
(restriction fragment length polymorphism and simple sequence repeat, 230 segregating loci), and a total of 23 linkage groups were produced, spanning 2,469 centi-Morgan (cM) (approximately $2 / 3$ of the whole genome of bread wheat and spelt) with an average marker density of $13.6 \mathrm{cM}$ (Messmer et al., 1999). Linkage analysis was repeated using JoinMap v4 (Van Ooijen, 2006), and the same genetic map was obtained, with slightly different total coverage. For QTL analysis, the mean value of a trait over replicates in each year was used. Interval mapping was carried out using MapQTL v6 to estimate the QTL locations, logarithm of the odds (LOD) scores, additive effects, and phenotypic variation explained by individual QTL $\left(\mathrm{R}^{2}\right)$ (Van Ooijen, 2009). A permutation test with 1,000 iterations was performed to estimate the genome-wide significance threshold $(\mathrm{P}<0.05)$. The markers closest to QTL peaks were selected as co-factors, and a set of significant co-factors $(\mathrm{P}<0.02)$ were used in the multiple-QTL model (MQM) mapping. QTL designations followed the Catalogue of Gene Symbols for Wheat (http://wheat.pw.usda.gov/GG2/Triticum/wgc/2008/). The 1-LOD (falloff from the QTL maximum LOD peaks) support intervals were calculated for QTL map positions, and drawn using MapChart v2.2 (Voorrips, 2002). Expression of the 'increasing' (increasing the trait values) and 'decreasing' (decreasing the trait values) alleles of QTL was used to analyse the allelic effects. 


\section{Results}

\subsection{Genetic variation in biomass and yield traits in the Forno $\times$ Oberkulmer mapping population}

Biomass accumulation and partitioning at key growth stages (GS39, anthesis and maturity), leaf area, plant height, and yield components, were analysed for phenotypic variation (Supplementary Table S1). As expected, the spelt Oberkulmer usually accumulated more total biomass and accordingly more mass for each plant organ (leaves, stems and spikes) at each stage than bread wheat Forno, but showing lower HI and similar grain weight per spike. Higher leaf area, lower SFI, taller plants and faster spike growth from GS39 to anthesis were also found in Oberkulmer. The RILs derived from Forno $\times$ Oberkulmer showed large variation and transgressive segregation in all traits (Supplementary Table S1). $\mathrm{H}^{2}$ differed greatly among traits: TGW, grains per spike, spike DW at different stages (spike $=$ chaff at maturity), spike:structural stem, total leaf and stem DW at maturity, and plant height, had relatively high $\mathrm{H}^{2}(>0.70)$, whereas the traits such as HI, grain weight per spike and CGR had low $\mathrm{H}^{2}(<0.40)$ (Supplementary Table S1).

\subsection{Relationships of biomass and leaf green area at GS39 with yield components}

At GS39, total DW per shoot was strongly associated with total leaf and stem DW, rather than spike DW (Supplementary Table S2), indicating that the biomass accumulated at this stage was mainly used to grow leaves and stems. In addition, total DW per shoot showed no or weak relationships with the percentage leaf and stem DW, suggesting that more total biomass did not change the dry-matter proportions of individual organs . A negative relationship between total DW per shoot and percentage spike DW was observed, so more total biomass could slightly reduce the proportion of spike DW. Among plant organs, leaf and stem DW were positively correlated, but their percentage partitioning negatively correlated. In contrast, spike DW and its percentage partitioning were weakly correlated with that of leaves and 
stems, indicating relatively independent growth at this stage. Total leaf area showed a close relationship with total DW per shoot at GS39 in both years $(\mathrm{r}=0.84, \mathrm{P}<0.01$ in $2012 ; \mathrm{r}=$ $0.82, \mathrm{P}<0.01$ in 2013).

Biomass accumulation and partitioning at GS39 were associated with yield components (Table 1). Total DW per shoot at this stage showed positive relationships with TGW, grains per spike, final biomass, and, in turn, grain weight per spike. The effects of leaf DW on yield components depended on years: it contributed to TGW in 2012, but to grains per spike in 2013. However, more leaf DW was associated with higher final biomass and grain weight per spike in both seasons. Stem DW consistently contributed to TGW, final biomass and grain weight per spike. Spike DW, on the other hand, appeared not to affect grains per spike and other yield components. The percentage partitioning of biomass influenced yield components only in 2013: increased percentage leaf DW was associated with slightly lower TGW, more grains per spike, higher final biomass and grain weight per spike, whereas the percentage stem and spike DW had the opposite effects. Leaf area at GS39 showed similar relationships with yield components as leaf DW (Table 1).

Table 1 Correlations between biomass (dry weight, DW) and total leaf area at GS39 (full flag leaf emergence), and yield components.

\begin{tabular}{|c|c|c|c|c|c|c|c|c|c|c|}
\hline \multirow{2}{*}{$\begin{array}{l}\text { Biomass and total } \\
\text { leaf area at GS39 }\end{array}$} & \multicolumn{2}{|c|}{ Thousand grain weight } & \multicolumn{2}{|c|}{ Grains per spike } & \multicolumn{2}{|c|}{ DW per shoot at maturity } & \multicolumn{2}{|c|}{ Harvest index } & \multicolumn{2}{|c|}{ Grain weight per spike } \\
\hline & 2012 & 2013 & 2012 & 2013 & 2012 & 2013 & 2012 & 2013 & 2012 & 2013 \\
\hline Total leaf DW & $0.38 * *$ & 0.00 & 0.00 & $0.51 * *$ & $0.50 * *$ & $0.74 * *$ & -0.14 & -0.17 & $0.25^{*}$ & $0.59 * *$ \\
\hline Stem DW & $0.43 * *$ & $0.26^{* *}$ & 0.11 & 0.17 & $0.52 * *$ & $0.59 * *$ & 0.06 & $-0.31 * *$ & $0.35 * *$ & $0.39 * *$ \\
\hline Spike DW & 0.13 & $0.28 * *$ & -0.03 & -0.17 & 0.12 & 0.00 & 0.14 & 0.03 & 0.18 & 0.02 \\
\hline DW per shoot & $0.43 * *$ & 0.18 & $0.24^{*}$ & $0.33 * *$ & $0.57 * *$ & $0.70 * *$ & 0.00 & $-0.27 * *$ & $0.42 * *$ & $0.51 * *$ \\
\hline Total leaf DW (\%) & 0.05 & $-0.28 * *$ & 0.22 & $0.52 * *$ & 0.20 & $0.39 * *$ & $-0.28 *$ & 0.08 & 0.19 & $0.38 * *$ \\
\hline Stem DW (\%) & 0.03 & $0.27 * *$ & 0.12 & $-0.48 * *$ & 0.15 & $-0.33 * *$ & 0.16 & -0.11 & 0.20 & $-0.35^{* *}$ \\
\hline Spike DW (\%) & -0.06 & 0.17 & -0.12 & $-0.38 * *$ & -0.17 & $-0.44 * *$ & 0.04 & $0.19 *$ & -0.06 & $-0.31 * *$ \\
\hline Total leaf area & $0.26^{*}$ & 0.10 & 0.02 & $0.44 * *$ & $0.38 * *$ & $0.67 * *$ & -0.19 & -0.05 & 0.20 & $0.59 * *$ \\
\hline
\end{tabular}

* Significant at $\mathrm{P}<0.05, * *$ significant at $\mathrm{P}<0.01$. 


\subsection{Relationships of biomass and leaf green area at anthesis with yield components}

At anthesis, total DW per shoot was closely correlated with the DW of each organ (Supplementary Table S3), but showed no or weak correlations with the percentage organ DW (Supplementary Table S4), indicating relatively proportional growth. Plant organ DW was positively correlated with each other (Supplementary Table S3). In terms of the percentage DW partitioning, there were no or positive relationships between leaves and spikes (Supplementary Table S4), but both were strongly and negatively correlated with total stems. A strong negative relationship between the percentage partitioning of WSCs and structural stems was found. Again, total leaf area and DW per shoot at anthesis were positively associated in both years $(\mathrm{r}=0.74, \mathrm{P}<0.01$ in both 2012 and 2013).

Higher DW of the whole shoot and each organ was associated with increased TGW in 2012, grains per spike in 2013, final biomass and grain weight per spike in both years, with the exceptions of stem WSC content and spike DW, which contributed to TGW and grains per spike consistently across years, respectively (Table 2). To avoid the effect of differences in spikelet number on the relationship between spike DW and grains per spike, the spikelet DW and grains per spikelet were calculated, and still exhibited a positive relationship $(\mathrm{r}=0.35, \mathrm{P}$ $<0.01$ in 2012; $\mathrm{r}=0.64, \mathrm{P}<0.01$ in 2013). Furthermore, SFI was also positively associated with grains per spike $(\mathrm{r}=0.57, \mathrm{P}<0.01$ in $2012 ; \mathrm{r}=0.33, \mathrm{P}<0.01$ in 2013). Increased biomass (mainly structural stems) was associated with slightly reduced HI in 2013. Spike:structural stem showed positive relationships with grains per spike, HI and grain weight per spike, but a negative relationship with TGW. For the percentage partitioning of biomass, the increased proportion of flag leaf DW was associated with increased grains per spike, final biomass and grain weight per spike in 2013 (Table 2). Percentage stem DW (mainly WSCs) was positively associated with TGW, but negatively associated with grains per spike, whereas 
the opposite was true for percentage spike DW (SPI), resulting in increased or decreased grain weight per spike, depending on years.

Flag leaf area was positively correlated with grains per spike, final biomass and grain weight per spike in both years (Table 2). Remaining and total leaf area was positively correlated with TGW in 2012, grains per spike in 2013, final biomass and grain weight per spike in both years.

Table 2 Correlations between biomass (dry weight, DW) and leaf area at anthesis, and yield components.

\begin{tabular}{|c|c|c|c|c|c|c|c|c|c|c|}
\hline \multirow{2}{*}{$\begin{array}{l}\text { Biomass and leaf area } \\
\text { at anthesis }\end{array}$} & \multicolumn{2}{|c|}{ Thousand grain weight } & \multicolumn{2}{|c|}{ Grains per spike } & \multicolumn{2}{|c|}{ DW per shoot at maturity } & \multicolumn{2}{|c|}{ Harvest index } & \multicolumn{2}{|c|}{ Grain weight per spike } \\
\hline & 2012 & 2013 & 2012 & 2013 & 2012 & 2013 & 2012 & 2013 & 2012 & 2013 \\
\hline Flag leaf DW & $0.32 * *$ & -0.02 & 0.09 & $0.54 * *$ & $0.35^{* *}$ & $0.70 * *$ & 0.02 & -0.09 & $0.32 * *$ & $0.60 * *$ \\
\hline Remaining leaf DW & $0.49 * *$ & 0.07 & 0.18 & $0.40 * *$ & $0.48 * *$ & $0.68 * *$ & 0.06 & $-0.20^{*}$ & $0.34 * *$ & $0.53 * *$ \\
\hline Total leaf DW & $0.52 * *$ & 0.04 & 0.18 & $0.49 * *$ & $0.52 * *$ & $0.75 * *$ & 0.06 & -0.17 & $0.39 * *$ & $0.60 * *$ \\
\hline Stem DW & $0.53 * *$ & $0.22 *$ & 0.2 & 0.16 & $0.61 * *$ & $0.58 * *$ & 0.08 & $-0.41 * *$ & $0.40 * *$ & $0.34 * *$ \\
\hline Stem WSC ${ }^{a}$ & $0.55 * *$ & $0.20 *$ & 0.17 & -0.11 & $0.56^{* *}$ & 0.12 & 0.21 & -0.16 & $0.48 * *$ & 0.04 \\
\hline Structural stem DW & $0.39 * *$ & 0.14 & 0.16 & $0.26 * *$ & $0.49 * *$ & $0.62 * *$ & -0.01 & $-0.39 * *$ & $0.26^{*}$ & $0.38 * *$ \\
\hline Spike DW & $0.23 *$ & $-0.23^{*}$ & $0.32 * *$ & $0.69 * *$ & $0.51 * *$ & $0.70 * *$ & -0.09 & -0.05 & $0.32 * *$ & $0.62 * *$ \\
\hline Spike:structual stem & -0.22 & $-0.42 * *$ & $0.25^{*}$ & $0.59 * *$ & -0.01 & $0.26^{* *}$ & -0.14 & $0.29 * *$ & -0.01 & $0.37 * *$ \\
\hline DW per shoot & $0.51 * *$ & 0.09 & 0.21 & $0.39 * *$ & $0.62 * *$ & $0.72 * *$ & 0.05 & $-0.32 * *$ & $0.40 * *$ & $0.51 * *$ \\
\hline Flag leaf DW (\%) & -0.11 & -0.1 & -0.05 & $0.49 * *$ & -0.18 & $0.49 * *$ & -0.02 & 0.11 & -0.03 & $0.49 * *$ \\
\hline Remaining leaf DW (\%) & 0.21 & -0.03 & -0.03 & 0.12 & 0.07 & 0.09 & 0.05 & 0.14 & 0.09 & 0.15 \\
\hline Total leaf DW (\%) & 0.13 & -0.08 & -0.06 & $0.37 * *$ & -0.04 & $0.34 * *$ & 0.04 & 0.17 & 0.06 & $0.38 * *$ \\
\hline Stem DW (\%) & $0.27 *$ & $0.37 * *$ & -0.16 & $-0.66^{* *}$ & 0.19 & $-0.41 * *$ & 0.18 & $-0.26 * *$ & 0.09 & $-0.49 * *$ \\
\hline Stem WSC (\%) & $0.43 * *$ & 0.17 & -0.01 & $-0.32 * *$ & $0.37 * *$ & $-0.24 * *$ & $0.24 *$ & -0.02 & $0.39 * *$ & $-0.23 *$ \\
\hline Structural stem DW (\%) & $-0.23 *$ & 0.1 & -0.09 & -0.17 & -0.22 & -0.05 & -0.12 & -0.18 & $-0.24 *$ & -0.14 \\
\hline Spike DW (\%) & $-0.50 * *$ & $-0.47 * *$ & $0.27 *$ & $0.67 * *$ & -0.21 & $0.33^{* *}$ & $-0.28 *$ & $0.26^{* *}$ & $-0.32 * *$ & $0.42 * *$ \\
\hline Flag leaf area & 0.20 & -0.01 & $0.24 *$ & $0.51 * *$ & $0.25 *$ & $0.69 * *$ & 0.03 & -0.08 & $0.32 * *$ & $0.59 * *$ \\
\hline Remaining leaf area & $0.27 *$ & 0.01 & 0.07 & $0.47 * *$ & $0.42 * *$ & $0.65 * *$ & 0.05 & -0.07 & $0.27 *$ & $0.56 * *$ \\
\hline Total leaf area & $0.25^{*}$ & 0.00 & 0.10 & $0.53 * *$ & $0.41^{* *}$ & $0.72 * *$ & 0.05 & -0.08 & $0.27 *$ & $0.62 * *$ \\
\hline
\end{tabular}

${ }^{a}$ WSC, water soluble carbohydrate.

* Significant at $\mathrm{P}<0.05, * *$ significant at $\mathrm{P}<0.01$.

\subsection{Relationships of biomass partitioning at maturity with yield components}

More biomass per shoot at maturity was closely associated with higher DW of leaves, stems, grains and chaff (Supplementary Table S5). More total biomass did not affect the percentage partitioning of biomass to different organs in 2012, but was weakly associated with higher 
proportions of flag leaves and chaff, and with lower proportion of stems in 2013 (Supplementary Table S6). Positive relationships among plant organ DWs were found (Supplementary Table S5). For the percentage partitioning of biomass, there were no (in 2012) or positive (in 2013) relationships between leaves and chaff, but both were negatively correlated with stems (Supplementary Table S6).

Turning to yield components, the DW of leaves, stems and chaff was positively correlated with either TGW or grains per spike, resulting in higher grain weight per spike (Table 3). Higher DW and percentage partitioning of stems and chaff were associated with reduced HI.

Table 3 Correlations between biomass partitioning at maturity and yield components.

\begin{tabular}{|c|c|c|c|c|c|c|c|c|c|c|}
\hline \multirow{2}{*}{$\begin{array}{l}\text { Biomass at maturity } \\
\text { (DW, dry weight) }\end{array}$} & \multicolumn{2}{|c|}{ Thousand grain weight } & \multicolumn{2}{|c|}{ Grains per spike } & \multicolumn{2}{|c|}{ DW per shoot at maturity } & \multicolumn{2}{|c|}{ Harvest index } & \multicolumn{2}{|c|}{ Grain weight per spike } \\
\hline & 2012 & 2013 & 2012 & 2013 & 2012 & 2013 & 2012 & 2013 & 2012 & 2013 \\
\hline Total leaf DW & $0.48 * *$ & 0.09 & 0.08 & $0.55^{* *}$ & $0.71 * *$ & $0.87 * *$ & 0.03 & -0.17 & $0.45 * *$ & $0.71 * *$ \\
\hline Stem DW & $0.43 * *$ & $0.32 * *$ & 0.09 & $0.26^{* *}$ & $0.82 * *$ & $0.81 * *$ & -0.10 & $-0.50 * *$ & $0.38 * *$ & $0.52 * *$ \\
\hline Chaff DW & 0.10 & 0.04 & $0.37 * *$ & $0.60 * *$ & $0.56^{* *}$ & $0.87 * *$ & $-0.52 * *$ & $-0.19 *$ & $0.37 * *$ & $0.71 * *$ \\
\hline Total leaf DW (\%) & 0.00 & -0.09 & -0.14 & 0.14 & -0.16 & $0.26^{* *}$ & -0.10 & $-0.25 * *$ & -0.06 & 0.11 \\
\hline Stem DW (\%) & -0.18 & $0.21 *$ & -0.16 & $-0.63 * *$ & -0.13 & $-0.27 * *$ & $-0.34 * *$ & $-0.74 * *$ & $-0.35 * *$ & $-0.58 * *$ \\
\hline Chaff DW (\%) & $-0.25 *$ & -0.17 & $0.31 * *$ & $0.35 * *$ & 0.01 & $0.44 * *$ & $-0.71 * *$ & $-0.29 * *$ & $-0.51 * *$ & $0.27 * *$ \\
\hline
\end{tabular}

* Significant at $\mathrm{P}<0.05, * *$ significant at $\mathrm{P}<0.01$.

\subsection{Crop growth dynamics}

Dynamics of biomass accumulation and partitioning from GS39 to maturity were analysed (Fig. 1). Total biomass accumulation was rapid from GS39 to anthesis, but became slow thereafter. Only a slight increase in total leaf DW was found between GS39 and anthesis, during which stems grew fast; both decreased during grain filling. The percentage leaf DW decreased continuously, while that of stem DW levelled off at GS39 and then underwent a reduction after anthesis. Spikes were very small at GS33 (only $0.6 \%$ of spike DW at anthesis in 2012, and $0.7 \%$ in 2013) and GS39 (5.4\% in 2012 and 6.0\% in 2013), and then grew fast until anthesis. Among plant organs, only grain weight increased significantly after anthesis. Despite similar growth patterns, crops developed earlier in 2013 than in 2012. 

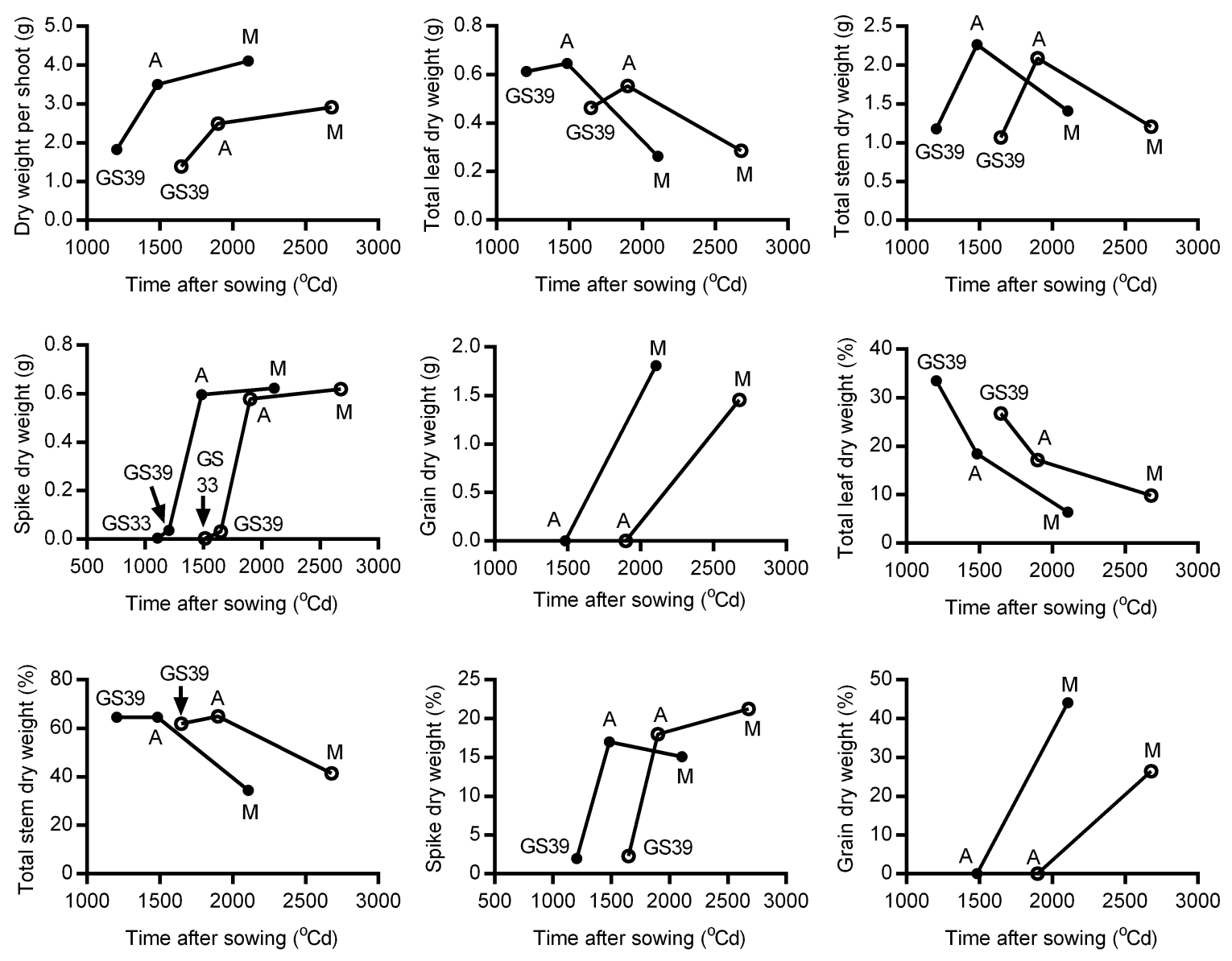

Fig. 1. Dynamics of biomass accumulation and partitioning. Data (means over replicates and across all lines) in 2012 and 2013 are shown as open and closed circles, respectively. GS33, the time when the third stem node is just detectable; GS39, the time at full flag leaf emergence; A, anthesis; M, maturity; ${ }^{\circ} \mathrm{Cd}$, degree days. 
It was found that the ADM and CGR from anthesis to maturity were only $32 \%$ and $12 \%$ of that from GS39 to anthesis, respectively (Supplementary Table S1). Correlation analysis showed that ADM and CGR between GS39 to anthesis were positively associated with spike DW at anthesis, TGW (only in 2012), grains per spike (only in 2013), final biomass and grain weight per spike in both years (Table 4). Similarly, ADM and CGR during grain filling were positively associated with grains per spike in 2013, and with TGW, final biomass and grain weight per spike in both years.

As the spikes grew mainly from GS39 to anthesis, only the SGR during this period was analysed (Supplementary Table S1). Spike DW at anthesis was strongly associated with SGR, rather than the duration from GS39 to anthesis (Table 4). SGR contributed to TGW in 2012, grains per spike in 2013, final biomass and grain weight per spike in both years (Table 4).

Table 4 Correlations between crop and spike growth, and yield components.

\begin{tabular}{|c|c|c|c|c|c|c|c|c|c|c|c|c|}
\hline \multirow{2}{*}{$\begin{array}{l}\text { Crop and spike } \\
\text { growth trait }\end{array}$} & \multicolumn{2}{|c|}{ Spike DW (A) } & \multicolumn{2}{|l|}{ TGW } & \multicolumn{2}{|c|}{ Grains per spike } & \multicolumn{2}{|c|}{ DW per shoot $(\mathrm{M})$} & \multicolumn{2}{|l|}{$\mathrm{HI}$} & \multicolumn{2}{|c|}{ Grain weight per spike } \\
\hline & 2012 & 2013 & 2012 & 2013 & 2012 & 2013 & 2012 & 2013 & 2012 & 2013 & 2012 & 2013 \\
\hline ADM (GS39-A) & $0.74 * *$ & $0.63 * *$ & $0.38 * *$ & -0.02 & 0.10 & $0.29 * *$ & $0.40 * *$ & $0.46^{* *}$ & -0.01 & $-0.23 *$ & $0.28^{*}$ & $0.31 * *$ \\
\hline CGR (GS39-A) & $0.62 * *$ & $0.44 * *$ & $0.43 * *$ & 0.09 & -0.04 & 0.12 & $0.31 * *$ & $0.31 * *$ & 0.08 & $-0.21 *$ & $0.23^{*}$ & $0.19 *$ \\
\hline $\operatorname{ADM}(\mathrm{A}-\mathrm{M})$ & 0.12 & 0.13 & $0.31 * *$ & $0.20 *$ & 0.13 & $0.49 * *$ & $0.50 * *$ & $0.63 * *$ & -0.08 & $0.29 * *$ & $0.35 * *$ & $0.71 * *$ \\
\hline CGR (A-M) & 0.07 & 0.06 & $0.30 * *$ & $0.22 *$ & 0.10 & $0.45 * *$ & $0.51 * *$ & $0.60 * *$ & -0.04 & $0.29 * *$ & $0.31 * *$ & $0.69 * *$ \\
\hline SGR (GS39-A) & $0.87 * *$ & $0.95 * *$ & $0.34 * *$ & $-0.19 *$ & 0.14 & $0.64 * *$ & $0.43 * *$ & $0.65 * *$ & 0.02 & 0.00 & $0.30 * *$ & $0.59 * *$ \\
\hline
\end{tabular}

${ }^{a}$ Abbreviations of the traits: ADM, accumulated dry matter; CGR, crop growth rate; SGR, spike growth rate; GS39, the time at full flag leaf emergence; A, anthesis; M, maturity; DW, dry weight; TGW, thousand grain weight; HI, harvest index.

* Significant at $\mathrm{P}<0.05, * *$ significant at $\mathrm{P}<0.01$. 


\subsection{Responses of yield components to de-tillering at GS39}

De-tillering at GS39 slightly reduced TGW, but increased grains per spike, total DW per shoot, and grain weight per spike (Table 5). No significant effect on HI was observed.

Table 5 Effects of de-tillering at GS39 (full flag leaf emergence) on yield components.

\begin{tabular}{|c|c|c|c|c|c|c|}
\hline \multirow[b]{2}{*}{ Yield component } & \multicolumn{2}{|c|}{ Mean across five lines } & \multicolumn{3}{|c|}{ P-value (NS, not significant; *, $\mathrm{P}<0.05 ; * *, \mathrm{P}<0.01$ ) } & \multirow{2}{*}{$\begin{array}{l}\text { De-tillering } \\
\text { effect (\%) }\end{array}$} \\
\hline & Control & De-tillering & Treatment & Line & Treatment $\times$ line & \\
\hline Thousand grain weight (g) & 44.2 & 41.3 & $* *$ & $* *$ & NS & -6.6 \\
\hline Grains per spike & 39.9 & 46.9 & $* *$ & $* *$ & NS & +17.5 \\
\hline Dry weight per shoot (g) & 3.83 & 4.28 & $* *$ & $* *$ & NS & +11.7 \\
\hline Harvest index & 0.46 & 0.44 & NS & * & NS & NS \\
\hline Grain weight per spike (g) & 1.75 & 1.91 & * & $* *$ & NS & +9.1 \\
\hline
\end{tabular}

\subsection{Responses of individual grain weight and dimensions to de-graining at anthesis}

De-graining at anthesis only slightly increased TGW (7.8\%) (Fig. 2 and Table 6). Grain morphological analysis showed that the treated RILs had higher grain width, height, and volume; however, this treatment did not affect grain length, and even reduced grain density. Furthermore, the RILs with smaller and more grains tended to increase TGW more than the others (Fig. 2).

Table 6 Effects of de-graining at anthesis on thousand grain weight (TGW, 112 lines used) and grain dimensions (10 lines used).

\begin{tabular}{|c|c|c|c|c|c|c|}
\hline \multirow[b]{2}{*}{ Trait } & \multicolumn{2}{|c|}{ Mean across the lines used } & \multicolumn{3}{|c|}{ P-value (NS, not significant; * $\mathrm{P}<0.05 ; * *, \mathrm{P}<0.01$ ) } & \multirow{2}{*}{$\begin{array}{l}\text { De-graining } \\
\text { effect (\%) }\end{array}$} \\
\hline & Control & De-graining & Treatment & Line & Treatment $\times$ line & \\
\hline TGW (g) & 45.0 & 48.5 & $* *$ & $* *$ & $* *$ & +7.8 \\
\hline Grain length (mm) & 7.4 & 7.5 & NS & ** & $*$ & NS \\
\hline Grain width (mm) & 3.2 & 3.5 & $* *$ & $* *$ & $*$ & +9.4 \\
\hline Grain height (mm) & 2.8 & 3.0 & $* *$ & $* *$ & NS & +7.1 \\
\hline Grain volume $\left(\mathrm{mm}^{3}\right)$ & 35.8 & 41.1 & $* *$ & $* *$ & NS & +14.8 \\
\hline Grain density $\left(\mathrm{g} \mathrm{cm}^{-3}\right)$ & 1.26 & 1.17 & $* *$ & NS & NS & -7.1 \\
\hline
\end{tabular}



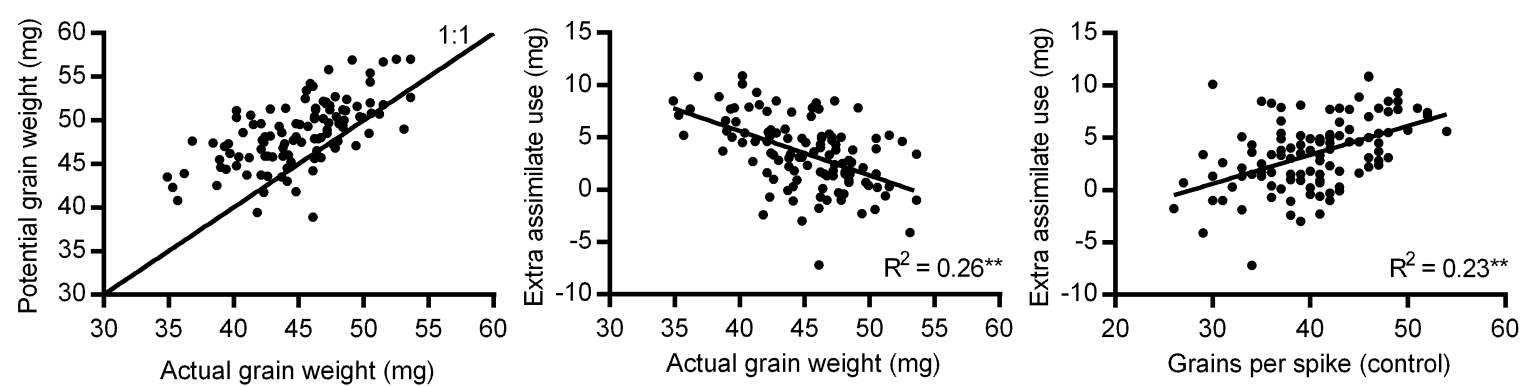

Fig. 2. Grain weight response to de-graining at anthesis. Actual grain weight, the individual grain weight of control spikes (intact); potential grain weight, the individual grain weight of trimmed spikes; extra assimilate use, the difference between potential and actual grain weight. For regression analysis: $* \mathrm{P}<0.05 ; * * \mathrm{P}<0.01$.

\subsection{Relationships of plant height with yield components}

Plant height was weakly correlated with final biomass, and there were some RILs having short plants but relatively high biomass (Fig. 3). Plant height was not associated with the other yield traits except a negative relationship with $\mathrm{HI}$ in $2013(\mathrm{r}=-0.60, \mathrm{P}<0.01)$.

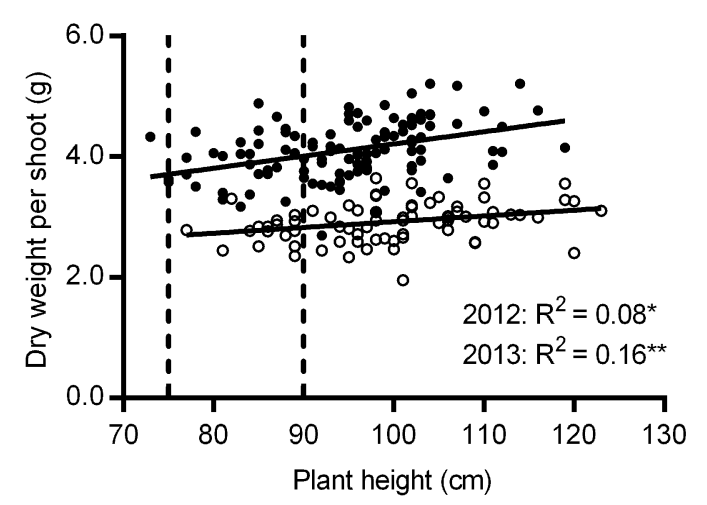

Fig. 3. Relationship between plant height and dry weight per shoot at maturity. Data in 2012 and 2013 are shown as open and closed circles, respectively. Vertical dashed lines indicate the range of plant height of wheat cultivars currently used in the UK. For regression analysis: * $\mathrm{P}<0.05$; ** $\mathrm{P}<0.01$. 


\subsection{QTL for biomass and biomass-related traits, and the QTL coincidences with those for yield components}

For biomass accumulation and partitioning, 156 QTL were detected at GS39, anthesis and maturity, individually explaining $11.3-33.9 \%$ of the phenotypic variation (Fig. 4 and Supplementary Table S7). Ten QTL for total DW per shoot across different stages were found on chromosomes 4A, 4B, 5A, and 5B; nine of them were coincident with 37 QTL for the DW of leaves, stems and spikes, with all increasing alleles from the same parents, consistent with the strong positive relationships between total biomass and plant organs. Most QTL for the DW of leaves (flag and remaining leaves at anthesis and maturity), stems (WSCs and structural stems at anthesis), and spikes (spikelets at anthesis and chaff at maturity) were usually coincident, and their increasing alleles came from the same parents, explaining the positive relationships among them. Furthermore, most QTL for the percentage partitioning of biomass to different organs were also coincident, mainly on $1 \mathrm{BS}, 2 \mathrm{~B}, 5 \mathrm{~A}$, and $7 \mathrm{~B}$; the increasing alleles for leaves and spikes were from the same parents (with the exceptions of some QTL for spike DW at GS39), which usually conferred the decreasing alleles for stems.

The QTL coincidences between biomass and yield traits were also observed (Fig. 4). Specifically, 48 QTL for total DW per shoot and plant organ DW at GS39 and anthesis were coincident with 14 QTL for TGW, nine for grains per spike, three for final biomass, one for HI, and two for grain weight per spike; the increasing alleles were usually conferred by the same parents, except those located on 3B, 4A and 5A, where the negative QTL coincidences between TGW and grains per spike were present. There were five and two QTL detected for spike DW at anthesis and SFI, respectively, and all of them were coincident with those for grains per spike. Ten QTL for SPI were identified, and half of them coincident with grains per spike. The increasing alleles of these coincident QTL were conferred by the same parents, confirming the positive relationships between spike DW, SFI, SPI, and grains per spike. At 
maturity, 18 QTL for the DW of leaves, stems and chaff were also coincident with six QTL for TGW, four for grains per spike, and two for grain weight per spike.

A total of 21 QTL for leaf area at GS39 and anthesis were found (Fig. 4 and Supplementary Table S7). Of them, 19 QTL were coincident with those for the DW of whole shoots and plant organs at GS39 and anthesis, while 17 were coincident with those for yield components. The parents conferring increasing alleles for leaf area also provided the increasing alleles for biomass at different stages, either TGW or grains per spike (but not both), and grain weight per spike, in line with their positive phenotypic relationships.

One QTL for the ADM from GS39 to anthesis was identified on 5B, coincident with those for total DW and leaf area at anthesis (Fig. 4 and Supplementary Table S7). Five QTL for SGR were identified on 2B, 2D, 5A, and 7B, coincident with five QTL for spike DW at anthesis, one for TGW, six for grains per spike, one for final biomass, one for HI, and one for grain weight per spike. The increasing alleles originated from the same parents, except those for TGW and $\mathrm{HI}$ on $5 \mathrm{~A}$.

After de-graining at anthesis, one QTL for PGW was identified on 2A, coincident with one QTL for TGW (Fig. 4 and Supplementary Table S7). Additionally, two QTL for extra assimilate use were found on 7B, coincident with two QTL for grains per spike (with the increasing alleles from Oberkulmer) and two for TGW (with the increasing alleles from Oberkulmer and Forno, respectively), confirming that the RILs with more and smaller grains were more responsive to increased assimilate availability.

Two QTL for plant height were identified on 2A, respectively (Fig. 4 and Supplementary Table S7), and no QTL coincidence with yield components was found. 